\title{
La comprensión lectora y el rendimiento escolar"
}

\author{
MIGUEL ÁNGEL GARCÍA GARCÍA* \\ MAYRA ALEJANDRA ARÉVALO DUARTE \\ CÉSAR AUGUSTO HERNÁNDEZ SUÁREZ ${ }^{* * *}$
}

Recepción: 17 de octubre de 2017

Aprobación: 28 de enero de 2018

Forma de citar este artículo: García M. A., Arévalo M. A. \& Hernández C. A. (2018). La comprensión lectora y el rendimiento escolar. Cuadernos de Lingüística Hispánica, (32), 155- 174.

* Artículo de investigación, proviene del proyecto "La comprensión lectora: TIC en el contexto escolar" de la Maestría en Educación de la Universidad de Pamplona, avalada por el Grupo de Investigación en Pedagogía y Prácticas Pedagógicas (GIPEPP) en la Línea de Investigación Lenguaje y Saberes.

** Mg. Educación y TIC, Universidad de Salamanca, España. Docente Universidad Francisco de Paula Santander, Cúcuta, Colombia. Grupo de Investigación en Pedagogía y Prácticas Pedagógicas (GIPEPP). Línea de Investigación Lenguaje y Saberes. Correo electrónico: miguelangelgg@ufps.edu.co

*** Doctora en Educación y TIC, Universidad de Salamanca, España. Docente Universidad Francisco de Paula Santander, Cúcuta, Colombia. Correo electrónico: mayraarevalo@ufps.edu.co

***** Magíster en Enseñanza de las Ciencias, Universidad Nacional Experimental de Táchira, Venezuela. Docente Universidad Francisco de Paula Santander, Cúcuta, Colombia. Correo electrónico: cesaraugusto@ufps.edu.co 


\section{Resumen}

Este escrito pretende determinar las posibles relaciones entre el nivel de comprensión lectora de los estudiantes y su desempeño académico. Se realiza un estudio descriptivo y un análisis estadístico correlacional. Se aplicó el instrumento Prueba de Competencia Lectora para Educación Secundaria (CompLEC) a estudiantes de ambos sexos con edades comprendidas entre los 13 y 14 años del grado noveno de educación básica de una institución educativa pública. A partir del análisis descriptivo, los estudiantes presentan dificultades en el orden textual, inferencial y contextual; en el análisis correlacional de los datos de las diferentes asignaturas (Ciencias Naturales, Ciencias Sociales, Lengua Castellana y Matemáticas) tienen una correlación significativa positiva, pero no significa necesariamente la existencia de una relación de causa-efecto.

Palabras clave: comprensión lectora, rendimiento escolar, estrategias de aprendizaje.

\section{Reading Comprehension and School Performance}

\section{Abstract}

This study is aimed to determine the relationships between the level of reading comprehension of the students and their academic performance. A descriptive study and a correlational statistical analysis are carried out. The Reading Competency Test for High School was applied to students of both sexes among ages of 13 and 14 of the ninth grade of basic education of a public educational institution. From the descriptive analysis the students present difficulties at a textual, inferential and contextual level; in the correlational analysis of the data of the different subjects (natural sciences, social sciences, Spanish language and mathematics) they have a positive significant correlation, but it does not necessarily mean the existence of a cause-effect relationship.

Keywords: reading comprehension, school performance, learning strategies.

\section{La compréhension de lecture et la réussite scolaire}

\section{Résumé}

Cet écrit vise à déterminer les possibles rapports entre le niveau de compréhension de lecture des apprenants et leurs résultats académiques. On a réalisé une étude descriptive et une analyse statistique de corrélation. On a utilisé l'instrument Épreuve de Compétence de Lecture pour Éducation Secondaire (CompLEC) avec des élèves de deux

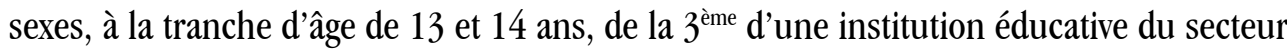


publique. À partir de l'analyse descriptive, les apprenants présentent des difficultés concernant l'ordre textuel, inférentiel et contextuel. Dans l'analyse de corrélation des données de différents cours (Sciences Naturelles, Sciences Sociales, Espagnol et Mathématiques) ils ont une corrélation significative positive, mais cela ne montre pas forcément l'existence d'un rapport de cause-effet.

Mots clés: compréhension de lecture, réussite scolaire, stratégies d'apprentissage.

\section{Compreensão em Leitura e Desempenho Escolar}

\section{Resumo}

Este artigo tem como objetivo determinar as possíveis relações entre o nível de compreensão de leitura dos alunos e seu desempenho acadêmico. Um estudo descritivo e uma análise estatística correlacional são realizados. 0 instrumento do Teste de Competência de Leitura para o Ensino Secundário (CompLEC) foi aplicado a estudantes de ambos os sexos com idades entre 13 e 14 anos do nono ano do ensino meio de uma instituição pública de ensino. A partir da análise descritiva, os alunos apresentam dificuldades na ordem textual, inferencial e contextual; na análise correlacional dos dados das diferentes disciplinas (Ciências Naturais, Ciências Sociais, Língua Espanhola e Matemática), elas têm uma correlação positiva significativa, mas não significa necessariamente a existência de uma relação de causa e efeito.

Palavras-chave: compreensão de leitura, desempenho escolar, estratégias de aprendizagem. 


\section{Introducción}

La sociedad actual se caracteriza por interpretar o producir textos y contenidos. El desenvolvimiento dentro del orden social, político, cultural y demás ámbitos se encuentra mediado por la capacidad para utilizar códigos y competencias para manejar el carácter especializado de los conocimientos. En otras palabras, el ejercicio de la comprensión lectora es una práctica cotidiana e imperativa en la configuración del mundo contemporáneo. De este modo, los aprendizajes autónomos de la sociedad del conocimiento a lo largo de la vida de un individuo se fundamentan en la comprensión de lectura. Más aun, cuando los conocimientos propios de los ciclos básicos de formación son insuficientes y deben ser actualizados constantemente debido al carácter cognitivo y a las transformaciones continúas que han asumido la mayoría de las actividades de la sociedad actual.

El propósito inherente de la lectura es la comprensión. Lo cual supone la capacidad del lector de disponer y utilizar diferentes estrategias que le ayuden a comprender distintos textos, intenciones textuales, objetivos de lectura y a resolver múltiples situaciones para hacer propio un determinado contenido. Se resalta que la comprensión es un proceso de carácter estratégico (Nieto, 2006; Muñoz \& Ocaña, 2017).

En este sentido, Solé (2012) afirma que el proceso de lectura debe asegurar la comprensión a través de la puesta en marcha de diferentes acciones que permitan a quien lee activar sus conocimientos previos para construir ideas sobre el contenido, organizar la información relevante, detenerse cuando lo requiera e incluso regresar y reflexionar; lo que implica espacios para desarrollar una lectura individual sin eliminar la posibilidad de concurrencias de diálogo y discusión. En este orden de ideas, la lectura en contextos académicos ha cobrado mayor importancia y está llamada a participar cada vez más activamente de los procesos de aprendizaje continuo y aprendizaje para la vida.

En cuanto a la definición de lectura, Solé (2012) sostiene que una definición más compleja de la lectura tiene más posibilidades de ser integral. Puesto que entender la lectura como simple decodificación es limitar los fines y los efectos que la lectura tiene en el aprendizaje. De acuerdo con esto, la OCDE (2006, p.48) entiende la lectura como "la capacidad de comprender, utilizar y analizar textos escritos, para alcanzar los objetivos del lector, desarrollar sus conocimientos y posibilidades y participar en la sociedad". Se trata de una definición más amplia que implica leer para varios fines, establecer estrategias de lectura que comprenden la comprensión y la autorregulación. Así mismo, se hace énfasis en la posibilidad que ofrece la lectura para que el sujeto-lector se desenvuelva y participe en la sociedad activamente. 
La lectura, como una tarea compleja, requiere procedimientos, secuencias de acciones y procesos que deben ser encaminados intencionalmente y armonizados con habilidades, destrezas y acciones cognitivas para lograr la construcción de significados y la comprensión. Por tanto, la lectura supone por definición una actividad estratégica que afecta la comprensión; por eso es necesario saber cuáles son las estrategias que afectan en mayor grado el nivel de comprensión, cuál es el uso que hacen los estudiantes de las estrategias en relación con su nivel de comprensión, y cómo se relaciona la comprensión y el rendimiento académico.

Si bien las investigaciones adelantadas frente a la comprensión lectora muestran evidencia teórica concluyente que sostiene resultados positivos en la aplicación de estrategias didácticas encaminadas a desarrollar la comprensión (Muñoz \& Ocaña, 2017), el camino aún no se agota, puesto que, a pesar de los nuevos fundamentos teóricos, los estudiantes todavía no muestran mejores resultados (Ortiz, 2008).

Al respecto, escribe Solé (2012) que existe la tendencia equivocada a pensar que

un estudiante, que ha aprendido a leer, podrá usar sin problemas la lectura para aprender a partir de distintos textos, como por ejemplo los textos disciplinares,-de elevada densidad léxica e informativa, de estructura expositiva, que presuponen conocimiento específico, que pueden presentar perspectivas distintas sobre una misma cuestión. (p. 52)

Los resultados de las investigaciones y las pruebas internacionales niegan constantemente este supuesto y revelan que la competencia lectora no se aplica indefinidamente, ni que existen estrategias que el estudiante maneja de manera innata, sino que es preciso participar en experiencias significativas que permitan al lector modelar, aprehender nuevas estrategias para asumir dichas situaciones.

Queda claro que no se nace con la habilidad de leer y que su aprendizaje requiere un incomparable esfuerzo en tiempo y dedicación (Sweet \& Snow, 2003). Desarrollar la comprensión lectora, por lo tanto, exige un proceso complejo que no descansa exclusivamente en las estrategias de comprensión. Existen otras dimensiones, aspectos que afectan la tarea del lector, más aun cuando la lectura responde a cambios culturales y construcciones sociales, económicas y tecnológicas, entre otras (Ferreiro, 2002; Cassany, 2006).

Vale recordar que dentro de las funciones principales de la educación básica secundaria establecidas por la Ley General de Educación de 1994 está la de formar estudiantes capaces de comprender textos, tarea aún pendiente. En este sentido, la experiencia de la práctica del aula permite evidenciar las deficiencias en cuanto a comprensión lectora por 
parte de los estudiantes y la falta de claridad de los mismos docentes a la hora de desarrollarla en ellos (Sánchez, García \& Rosales, 2010).

Solé (2012) plantea que existe una relación estrecha entre la competencia lectora y el aprendizaje; concomitante a la comprensión y como condición de esta, el uso de estrategias participa del proceso de construcción de significados que debe culminar en nuevos aprendizajes. En el contexto escolar, según las investigaciones, hay muchas variables predictivas del rendimiento académico, como la motivación, la responsabilidad y la personalidad, el estrato económico, el tiempo de dedicación, el uso de estrategias de estudio, altas expectativas, entre otras (Caso-Niebla \& Hernández-Guzmán, 2007).

Dentro de este abanico de variables, este escrito se centra y analiza la relación que se plantea entre el nivel de comprensión y el rendimiento académico. No se trata de una relación evidente, dado que en el caso del rendimiento académico, depende de aquello que los docentes consideren importante aprender y evaluar en sus asignaturas, puesto que no todos los maestros tienen en cuenta los mismos criterios, ni se orientan en la práctica bajo las mismas perspectivas pedagógicas, metodológicas y didácticas. A pesar de esta variabilidad, mucho de lo que se hace en el aula recurre a la lectura como medio de aprendizaje y puede dar indicios del uso de estrategias y su influencia en la comprensión de lectura.

La importancia de reconocer la relación entre el nivel de comprensión y el rendimiento académico, a diferencia de otras variables predictivas propias del rendimiento académico, es que en este caso se pueden adelantar acciones de diseño pedagógico e implementación didáctica orientadas hacia el mejoramiento de la comprensión lectora, contrario a la dificultad de afectar variables tales como la condición socioeconómica, el nivel de escolaridad de los padres, entre otras.

El contexto de la educación básica y media evidencia que los docentes dan por sentada la capacidad de comprensión lectora de sus estudiantes, hecho que se contradice con la situación real. Del mismo modo, la revisión de los antecedentes muestra que existen casos en que los profesores no perciben con claridad la relación entre las estrategias de lectura (crítica) y la comprensión de los estudiantes, lo que agudiza los bajos resultados (Moreno, 2005; Solé, 2007; Sánchez, García \& Rosales, 2010; Avendaño, 2016).

Una revisión en Colombia de los trabajos sobre investigación en comprensión lectora permite encontrar experiencias, propuestas y prácticas académicas que dan cuenta de la relevancia que ha venido adquiriendo el desarrollo de la comprensión 
lectora en el país. En el ámbito universitario se evidencia un esfuerzo comprometido de sistematización y de implementación de propuestas (May et al., 2001; Barletta, 2001; López \& Arciniegas, 2003; Perilla, Rincón, Gil \& Salas, 2004; Grupo Heterolalia, 2007; Grupo de Investigación DiLeMa, 2008a, 2008b; Galindo \& Moreno, 2014; Serna \& Díaz, 2015). En cuanto a la educación secundaria y media, no existen abundantes publicaciones sobre el tema. A pesar de ello, las investigaciones encontradas superan el simple diagnóstico y presentan propuestas y estrategias interesantes encaminadas a mejorar la comprensión, como la realizada por Muñoz y Ocaña (2017), y la enseñanza del ensayo argumentativo (Ochoa \& García, 2013).

\section{Método}

\subsection{Diseño y naturaleza de investigación}

El nivel de este estudio corresponde a una investigación cuantitativa, y el diseño utilizado es una investigación no experimental (ex post facto) de tipo correlacional. Las variables que se correlacionan en este estudio son las siguientes: comprensión de lectura y rendimiento académico. El tipo de coeficiente correlacional que se utilizó fue el Pearson (r), el cual es pensado para variables cuantitativas, y es un índice que mide el grado de covariación entre distintas variables relacionadas linealmente.

\subsection{Población y muestra}

La población corresponde a 97 estudiantes del grado noveno de educación básica de la Institución Julio Pérez Ferrero (Cúcuta, Colombia), que no superan los 14 años. La muestra quedó conformada por 91 estudiantes (65 de la mañana y 26 de la tarde; 47 niños y 44 niñas), que corresponde al $93.8 \%$ de la población. El porcentaje restante pertenece al número de estudiantes que no asistió el día de la aplicación de los instrumentos o que fueron excluidos porque se evidencia que llenaron la prueba sin leer o respondieron al azar. Todos los estudiantes que presentaron la prueba cumplen el rango de 13-14 años. Se trata de una muestra intencional, porque los criterios para su selección están enmarcados en la disponibilidad de los estudiantes y los espacios como recursos con los que se pudo contar para la investigación.

\subsection{Variables}

Comprensión de lectura. Se mide a través de tres niveles de comprensión: nivel textual, que se refiere a la capacidad de identificar la información. El segundo nivel, denominado inferencial, mide la capacidad de establecer relaciones entre las ideas, y el nivel contextual, relacionado con la valoración y validación de la información a partir del con- 
texto (Tabla 1). La variable de comprensión se evalúa a través del instrumento de lectura (CompLec), (Llorens et al., 2011).

Tabla 1. Variable comprensión de lectura.

\begin{tabular}{|c|c|c|c|}
\hline Variable 1 & Nivel & Componente & $\begin{array}{c}\text { Distribución de } \\
\text { preguntas CompLec }\end{array}$ \\
\hline \multirow{3}{*}{$\begin{array}{l}\text { Comprensión } \\
\text { de lectura }\end{array}$} & Nivel textual: literalidad & $\begin{array}{l}\text { Habilidad de ubicar e identificar } \\
\text { la información }\end{array}$ & $2,5,7,14,15$ \\
\hline & $\begin{array}{l}\text { Nivel inferencial: integración, } \\
\text { interpretación, construcción del } \\
\text { significado }\end{array}$ & $\begin{array}{l}\text { Capacidad de establecer rela- } \\
\text { ción entre las ideas }\end{array}$ & $\begin{array}{l}4,8,9,10,11,13,16 \\
17,18,20\end{array}$ \\
\hline & $\begin{array}{l}\text { Nivel contextual: validez, } \\
\text { reflexión y valoración }\end{array}$ & $\begin{array}{l}\text { Capacidad de analizar de forma } \\
\text { crítica el contenido }\end{array}$ & $1,3,6,12,19$ \\
\hline
\end{tabular}

Rendimiento académico. Se obtuvo a partir del promedio de la nota de los periodos I, II, y III del registro académico del año 2014, época en la que la mayoría de los estudiantes del estudio se encontraban cursando el grado octavo. Las asignaturas que se tienen en cuenta son: Lengua Castellana, Matemáticas, Ciencias Sociales y Ciencias Naturales. La escala de evaluación es de (1-5), donde de 1 a 2.9 es desempeño bajo, de 3 a 3.9 es desempeño básico; de 4 a 4.5 es desempeño alto y de 4.6 a 5 desempeño superior.

\section{Técnicas e instrumentos y análisis de la información}

El instrumento utilizado para la recolección de información es la "Prueba de Competencia Lectora para Educación Secundaria (CompLec)", (Llorens et al., 2011). Prueba creada para estudiantes entre 11 y 14 años, resultado de una investigación que arrojó un test de lectura baremado y con propiedades psicométricas de fiabilidad, homogeneidad y validez satisfactoria para el campo de la investigación. El instrumento, además de estar creado por especialistas, fue sometido a diferentes pruebas piloto en las que participaron en la aplicación definitiva un total de 1854 estudiantes.

La tabla de registro de notas recoge el nombre del estudiante, el género, el promedio de rendimiento académico de los tres primeros periodos en las asignaturas de Lengua Castellana, Matemáticas, Ciencias Sociales y Ciencias Naturales (plataforma de la Institución "OVY"). Además, incluye el total de preguntas correctas por estudiante del instrumento CompLec, y detalla el resultado por estudiante de cada uno de los niveles de comprensión (recuperación, integración y contextualización). Así mismo, presenta información sobre la identificación de la clase de texto por parte del estudiante. 
Se utilizó el programa para análisis estadístico SPSS, para el análisis de correlación se empleó el coeficiente de Pearson (r), que puede calcularse en cualquier grupo de datos; sin embargo, la validez del test de hipótesis sobre la correlación entre las variables requiere en sentido estricto (Altman, 1997): a) que las dos variables procedan de una muestra aleatoria de individuos, b) que al menos una de las variables tenga una distribución normal en la población de la cual procede la muestra. Para el cálculo válido de un intervalo de confianza del coeficiente de correlación de $r$, ambas variables deben tener una distribución normal. La cuantificación de la fuerza de la relación lineal entre dos variables cuantitativas se estudia por medio del cálculo del coeficiente de correlación de Pearson (DawsonSaunders \& Trapp, 1996; Milton \& Tsokos, 2001; Martín \& Luna del Castillo, 1993). Dicho coeficiente oscila entre -1 y +1 . Un valor de -1 indica una relación lineal o línea recta positiva perfecta. Una correlación próxima a cero indica que no hay relación lineal entre las dos variables.

\section{Resultados, descripción y análisis de los resultados}

\subsection{Análisis comprensión lectora}

Tabla 2. Descriptivos de la comprensión lectora de las cuatro asignaturas.

\begin{tabular}{|l|c|c|c|c|c|c|}
\hline \multicolumn{1}{|c|}{ Asignaturas } & Mínimo & Máximo & Media & $\begin{array}{c}\text { Desviación } \\
\text { Estándar }\end{array}$ & $\begin{array}{c}\text { Sesgo } \\
\text { Estandarizado }\end{array}$ & $\begin{array}{c}\text { Curtosis } \\
\text { Estandarizada }\end{array}$ \\
\hline Biología & 1,9 & 4,2 & 2,99 & 0,53 & $-0,04$ & $-0,99$ \\
\hline Ciencias Sociales & 2,3 & 4,5 & 3,23 & 0,47 & 1,23 & 0.21 \\
\hline $\begin{array}{l}\text { Lengua Castella- } \\
\text { na }\end{array}$ & 1,4 & 4,5 & 3,01 & 0,64 & $-0,25$ & 0,22 \\
\hline Matemáticas & 1,9 & 4,5 & 3,17 & 0,55 & 1,38 & 0,02 \\
\hline
\end{tabular}

Como se puede observar en la Tabla 2 y apoyados en una escala calificativa de 0 a 5 (siendo 5 la nota máxima), en la asignatura de Lengua Castellana los estudiantes de la muestra presentan la calificación más baja. En cuanto a la calificación más alta, se observan promedios muy similares entre las diversas asignaturas. Además, Ciencias Naturales y Lengua Castellana presentan en los estudiantes de la muestra la calificación promedio más baja, y la asignatura de Ciencias Sociales es la que ofrece el promedio más alto.

Con el fin de determinar si la muestra proviene de una distribución normal, tanto el sesgo estandarizado como la curtosis estandarizada permiten verificar esta condición. Como se puede observar en las cuatro asignaturas, los valores obtenidos para estos esta- 
dísticos se encuentran dentro del rango [-2,2], lo que es evidencia suficiente para afirmar que los datos son provenientes de una distribución normal.

Lo anterior es verificable de forma gráfica (Figura 1) a través de la utilización de los diagramas de cajas y bigotes en donde los valores mínimo y máximo se ubican en los extremos de los bigotes, y los cuartiles se distribuyen horizontalmente para formar la caja con el fin de identificar cómo se distribuyen los datos alrededor del estadístico central (la mediana) vislumbrando su variabilidad. Por ejemplo, en el gráfico de la asignatura de Matemáticas, se observa que en la parte izquierda de la caja se aglutina la mayor parte de las puntuaciones. Ello indica que las calificaciones comprendidas entre el $25 \%$ y el $50 \%$ de la muestra presentan mayor variabilidad en comparación con las calificaciones comprendidas entre el $50 \%$ y el $75 \%$. Cuando se comparan los bigotes, el de la izquierda resulta ligeramente menor que el de la derecha , lo que indica que el $25 \%$ de las calificaciones más bajas están más densamente concentradas que el $25 \%$ de las calificaciones más altas.
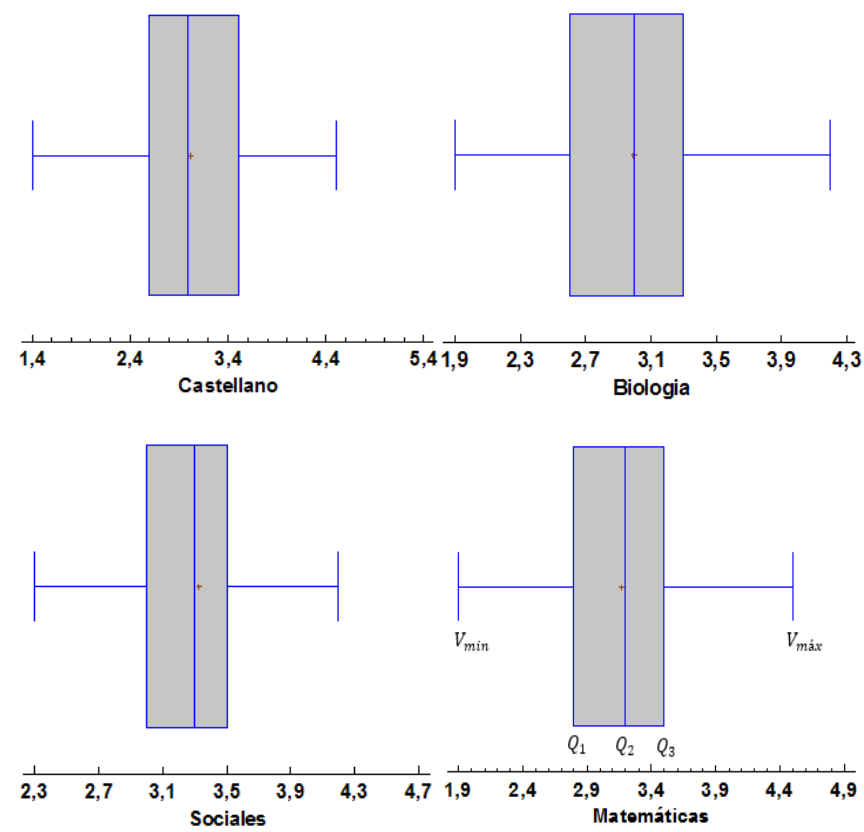

Figura 1. Diagramas de cajas y bigotes para verificar la normalidad de las asignaturas de Matemáticas, Ciencias Naturales, Lengua Castellana y Ciencias Sociales.

Un aspecto interesante para la investigación es determinar el nivel de respuestas correctas observables en los estudiantes de la muestra, dado que el instrumento CompLEC se compone de 20 ítems, se procedió a generar cuatro rangos para el número de respuestas acertadas (ver Tabla 3). 
Tabla 3. Rangos de respuestas acertadas de la aplicación del instrumento CompLEC

\begin{tabular}{|l|c|c|}
\hline \multicolumn{1}{|c|}{ Rango } & Frecuencia & Porcentaje \\
\hline De 1 a 5 correctas & 23 & 25,3 \\
\hline De 6 a 10 correctas & 60 & 65,9 \\
\hline De 11 a 15 correctas & 8 & 8,8 \\
\hline De 16 a 20 correctas & 0 & 0,0 \\
\hline Total & $\mathbf{9 1}$ & $\mathbf{1 0 0 , 0}$ \\
\hline
\end{tabular}

Como se puede observar en la Tabla 3, aproximadamente el $66 \%$ de los estudiantes evaluados respondió correctamente entre 6 y 10 ítems de la prueba aplicada. Cabe destacar que ningún estudiante acertó más de 15 ítems. Este resultado se identifica totalmente con los diagnósticos internacionales de las pruebas Pisa y los resultados nacionales de las pruebas Saber, que evidencian dificultades de los estudiantes en cuanto a la comprensión de lectura (Icfes, 2013). Sánchez, García y Rosales (2010) al respecto manifiestan que se ha avanzado mucho sobre lo que se puede hacer en el aula para mejorar la comprensión lectora, sin embargo, dichos avances no se han transferido a las prácticas de enseñanza aprendizaje.

A continuación se presenta el número de ítems resueltos correctamente en cada uno de los niveles de la comprensión lectora (ver Tabla 4).

Tabla 4. Número de aciertos por nivel de comprensión.

\begin{tabular}{|c|c|c|c|c|c|c|c|c|}
\hline \multicolumn{3}{|c|}{ Nivel textual o recuperación } & \multicolumn{3}{|c|}{ Nivel inferencial o de integración } & \multicolumn{3}{|c|}{ Nivel contextual } \\
\hline $\begin{array}{c}\mathbf{N} .^{\circ} \text { de } \\
\text { aciertos }\end{array}$ & $\mathbf{F r}$ & $\%$ & $\begin{array}{c}N .^{\circ} \text { de } \\
\text { aciertos }\end{array}$ & $\mathbf{F r}$ & $\%$ & $\begin{array}{c}\mathbf{N}^{\circ} \text { de } \\
\text { aciertos }\end{array}$ & $\mathbf{F r}$ & $\%$ \\
\hline 0 & 16 & 17,6 & 0 & 2 & 2,2 & 0 & 7 & 7,7 \\
\hline 1 & 22 & 24,2 & 1 & 6 & 6,6 & 1 & 13 & 14,3 \\
\hline 2 & 28 & 30,8 & 2 & 13 & 14,3 & 2 & 34 & 37,4 \\
\hline 3 & 19 & 20,9 & 3 & 26 & 28,6 & 3 & 23 & 25,3 \\
\hline 4 & 3 & 3,3 & 4 & 21 & 23,1 & 4 & 10 & 11,0 \\
\hline 5 & 3 & 3,3 & 5 & 16 & 17,6 & 5 & 4 & 4,4 \\
\hline Total & 91 & 100,0 & 6 & 3 & 3,3 & Total & 91 & 100,0 \\
\hline & & & 7 & 4 & 4,4 & & & \\
\hline & & & 8 & 0 & 0,0 & & & \\
\hline & & & 9 & 0 & 0,0 & & & \\
\hline & & & 10 & 0 & 0,0 & & & \\
\hline & & & Total & 91 & 100,0 & & & \\
\hline
\end{tabular}


De la Tabla 4, se destaca el alto número de estudiantes que no acertaron en ningún ítem de este primer nivel, que tal como se explicó en el marco teórico se refiere a la recuperación de información, esto es, resolver preguntas que se encuentran textualmente dentro de la lectura e identificar con diferentes términos lo mismo que dice el texto. Llama la atención igualmente el bajo número de estudiantes que demostraron dominio de los procesos asociadas a este nivel (4 y 5 ítems correctos).

Autores como Belmonte (2006) concuerdan en que para alcanzar la comprensión de lectura se llevan a cabo diferentes procesos que inician todos con la decodificación 0 nivel literal hasta la representación de niveles superiores en los que se crea, modifica, elabora e integra la representación de lo leído a la estructura del conocimiento del lector.

Frente al nivel inferencial, se evidencia que cerca del $84 \%$ de los estudiantes de la muestra respondió correctamente entre 2 y 5 ítems de un total de 10, lo que se manifiesta como una debilidad en las competencias asociadas a este nivel. Según OCDE (2006), Cisneros, Olave y Rojas (2012) y León (2003), en la construcción de inferencias, el lector, a partir de su experiencia complementa la información del texto a través de deducciones, conjeturas, supuestos y síntesis que el texto no explicita. Vale aclarar que la inferencia es el elemento imprescindible y eje generador de la comprensión, puesto que permite una aproximación al análisis de los subprocesos de la lectura (Borrero, 2008). Una comparación de los datos recogidos en la Institución con la revisión de la literatura evidencia la similitud en cuanto a la dificultad que presentan los estudiantes para realizar inferencias no solo en secundaria, sino también en la educación superior.

En el nivel de la lectura contextual, cabe destacar que aproximadamente el $60 \%$ de los estudiantes de la muestra se encuentra en el rango de 0 a 2 , y que un poco más del $4 \%$ contestó los cinco ítems. Lo cual evidencia una debilidad en la capacidad de saber qué hacer con los contenidos y los datos.

Frente a estos tres niveles de lectura (interpretación, inferencia y contextualización), Sánchez, García y Rosales (2010) aclaran que cada uno de los niveles de lectura aporta al proceso de comprensión y que no se dan de modo sucesivo o acumulativo sino que se interrelacionan, así que la existencia de un primer nivel no implica el éxito en el segundo o tercer nivel de comprensión. Es decir, que la correcta realización de un nivel no implica la superación del siguiente. Para nuestro estudio existe una tendencia significativa que evidencia que las dificultades se presentan en los tres niveles, mostrando mayor dificultad en el nivel inferencial y contextual. 
El instrumento utilizado CompLEC consta de cinco textos, cada uno de ellos configurado bajo una tipología textual específica, es decir, que a partir de la intencionalidad definida le corresponden unas características propias en cuanto a estructura. Lo cual ofrece mayores posibilidades de análisis del proceso de comprensión. Por ello, a continuación se presenta un informe descriptivo en donde se muestra si el estudiante identificó correctamente la estructura textual, lo cual según Solé (2007) y Van Dijk y Kintsch (1983) es fundamental en el proceso de comprensión lectora.

Tabla 5. Identificación de la estructura textual

\begin{tabular}{|c|c|c|c|c|c|c|c|c|c|c|}
\hline \multirow{2}{*}{$\begin{array}{c}\text { Clase de } \\
\text { Texto } \\
\text { Proceso }\end{array}$} & \multicolumn{2}{|c|}{$\begin{array}{c}\text { (Texto 1). El } \\
\text { calentamiento } \\
\text { global. (Texto } \\
\text { discontinuo- } \\
\text { expositivo) }\end{array}$} & \multicolumn{2}{|c|}{$\begin{array}{l}\text { (Texto 2). El } \\
\text { lenguaje de las } \\
\text { abejas. (Texto } \\
\text { Expositivo - } \\
\text { continuo) }\end{array}$} & \multicolumn{2}{|c|}{$\begin{array}{c}\text { (Texto 3). } \\
\text { Energía } \\
\text { nuclear (Texto } \\
\text { argumentativo- } \\
\text { continuo) }\end{array}$} & \multicolumn{2}{|c|}{$\begin{array}{c}\text { (Texto 4). } \\
\text { Accidentes de } \\
\text { tráfico. (Texto } \\
\text { discontinuo } \\
\text { informativo) }\end{array}$} & \multicolumn{2}{|c|}{$\begin{array}{c}\text { (Texto 5). } \\
\text { Siéntese en } \\
\text { sillas adecuadas. } \\
\text { (Texto expositivo } \\
\text {-continuo) }\end{array}$} \\
\hline & $\mathrm{Fr}$ & $\%$ & $\mathrm{Fr}$ & $\%$ & $\mathrm{Fr}$ & $\%$ & $\mathrm{Fr}$ & $\%$ & $\mathrm{Fr}$ & $\%$ \\
\hline Sí & 23 & 25,3 & 20 & 22,0 & 32 & 35,2 & 41 & 45,1 & 24 & 26,4 \\
\hline No & 68 & 74,7 & 71 & 78,0 & 59 & 64,8 & 50 & 54,9 & 67 & 73,6 \\
\hline Total & 91 & 100,0 & 91 & 100,0 & 91 & 100,0 & 91 & 100,0 & 91 & 100,0 \\
\hline
\end{tabular}

De la Tabla 5 se puede evidenciar que más del $60 \%$ de los estudiantes de la muestra presentó dificultades para identificar adecuadamente las características de los textos 1, 2, 3 y 5; de igual modo se presentan dificultades para relacionar la superestructura (Van Dijk \& Kintsch, 1983) y con ello problemas en la elección de estrategias para adelantar la lectura. Los textos (1, 2 y 5$)$ presentan características propias del texto expositivo y guardan un resultado semejante en cuanto a los bajos resultados que presentan los estudiantes para identificarlos. Lo cual sugiere precarios conocimientos en lo relativo a la estructura expositiva, debido a razones aún no precisadas que pueden o no estar relacionas con la ausencia de textos científicos en las prácticas de aula.

Autores como Coll (2005), afirman que la lectura en la sociedad de la información se convierte en una herramienta poderosa de producción de conocimiento y, en este sentido, Coiro (2003) explica que no es exactamente igual la lectura de un texto continuo, secuencial, tradicional a un texto discontinuo, puesto que este último aunque presenta información organizada, no lo hace necesariamente de forma progresiva y secuencial, y por tanto su lectura exige nuevas estrategias y recursos por parte del lector.

Si bien las estructuras textuales son independientes del contenido, influyen en la organización de este. Según Solé (2007), la activación de conocimientos previos referente a la organización del texto permite definir con mayor claridad qué esperar de este y, por 
tanto, cómo desarrollar mejor las estrategias que mejoran la comprensión, así mismo la estructura textual responde directamente a la intención del autor del texto.

\subsection{Análisis de correlación}

La correlación entre dos variables puede ser negativa o positiva. Se dice que existe correlación negativa entre dos variables si a medida que una aumenta, la otra disminuye y viceversa. Existe correlación positiva si al aumentar una variable la otra también aumenta o si disminuyen las dos. Hay que aclarar que el hecho de que dos variables estén correlacionadas positiva o negativamente, no es lo mismo que entre ellas exista una relación causa-efecto. Por ello, en esta investigación se busca determinar la posible existencia de correlación entre el nivel de desempeño en algunas áreas básicas de formación y el número de aciertos obtenidos por los estudiantes en la prueba CompLEC, a través de la aplicación del coeficiente de correlación de Pearson (ver Tabla 6).

Tabla 6. Coeficiente de correlación de Pearson prueba CompLEC y las áreas de formación básica.

\begin{tabular}{|l|c|c|c|c|c|}
\hline & Biología & $\begin{array}{c}\text { Ciencias } \\
\text { Sociales }\end{array}$ & $\begin{array}{c}\text { Lengua } \\
\text { castellana }\end{array}$ & Matemáticas & Comprensión \\
\hline Ciencias naturales & 1 & $0,4848 * *$ & $0,4750 * *$ & $0,5805 * *$ & $0,4574 * *$ \\
\hline Ciencias sociales & $0,4848 * *$ & 1 & $0,4571 * *$ & $0,5681 * *$ & $0,4448 * *$ \\
\hline Lengua castellana & $0,4750 * *$ & $0,4571 * *$ & 1 & $0,5471 * *$ & $0,4774 * *$ \\
\hline Matemáticas & $0,5805 * *$ & $0,5681 * *$ & $0,5471 * *$ & 1 & $0,4595 * *$ \\
\hline Comprensión & $0,4574 * *$ & $0,4448 * *$ & $0,4774 * *$ & $0,4595 * *$ & 1 \\
\hline
\end{tabular}

** La correlación es significativa al 1 \% (bilateral)

En la Tabla 6 se muestran las correlaciones momento de Pearson entre cada par de variables. El rango de estos coeficientes de correlación varía en el intervalo de $[-1,1]$ y miden la fuerza de relación lineal entre las variables. Se destacan los siguientes pares de variables: Biología y Ciencias Sociales, Biología y Lengua castellana, Biología y Matemáticas, Biología y Comprensión, Ciencias Sociales y Lengua Castellana, Ciencias Sociales y Matemáticas, Ciencias Sociales y Comprensión, Lengua castellana y Matemáticas, Lengua castellana y Comprensión, Matemáticas y Comprensión, ya que presentan correlaciones significativamente diferentes de cero y positivas; por lo tanto, según los datos obtenidos en esta muestra, si un estudiante obtiene buenos resultados en Biología, se esperaría que también obtenga buenos resultados en Ciencias Sociales o en Lengua Castellana o en Matemáticas o en Comprensión Lectora. 


\section{Discusión y conclusiones}

Frente al objetivo que busca establecer el nivel de comprensión lectora de los estudiantes y su posible relación con el desempeño escolar se encontró que en el nivel textual, en el cual se extrae información explícita del texto y se pregunta por el contenido, se evidencia una importante dificultad que muestra que aunque los estudiantes decodifican el texto, siguen aún sin entender a qué se refieren muchas de las ideas leídas y les cuesta expresar las afirmaciones del texto en sus propias palabras.

Sin embargo, las dificultades de comprensión lectora más importantes se concentran en el nivel inferencial y el nivel contextual. En el primero, el estudiante construye el significado y establece la relación de las ideas incluyendo información implícita, al realizar procesos de análisis, síntesis, inducción, deducción, etc. En este nivel se presenta mayor dificultad con problemas para deducir, analizar y concluir información nueva a partir de la que se encuentra en el texto. Esta debilidad ofrece una oportunidad clave para trabajar en procesos de inferencia y en estrategias de comprensión lectora.

En último término, el nivel contextual busca medir la capacidad para ubicar en un entorno de sentido la información del texto, lo cual significa en términos prácticos conocer la intención comunicativa del autor y de sus afirmaciones, valorar la utilidad del contenido, la validez de los argumentos, la pertinencia de la superestructura elegida y su coherencia con la intención comunicativa, así como analizar de forma crítica el contenido. Se evidencia, entonces, como posibilidad de mejoramiento, el desarrollo de la lectura crítica y el trabajo con estructuras textuales en orden a desarrollar estrategias de comprensión lectora pertinentes que no excluyan los procesos reflexivos, evaluativos, críticos y escriturales derivados (Avendaño, 2016).

Al revisar los porcentajes generales se evidencia que cerca del $90 \%$ de los estudiantes solamente resolvió correctamente menos de la mitad de las preguntas propuestas. Este mismo análisis permite establecer la consecuente correlación de los tres niveles de lectura analizados. Así mismo, estos resultados concuerdan con el nivel alcanzado en las pruebas Saber, grado 9, que ubican al 79 \% de los estudiantes de la Institución en el 2014 en los niveles de insuficiente y mínimo, y al $0 \%$ de estudiantes en el nivel avanzado (Documento MEN- Reporte de Excelencia 2015, Día de la Excelencia, para el establecimiento educativo Julio Pérez Ferrero).

Frente a los datos recogidos de rendimiento académico de los estudiantes en las asignaturas elegidas, el análisis estadístico establece la existencia de una correlación significativa positiva, lo que significa que los datos de las diferentes materias tienen una 
covariación conjunta y próxima, es decir, que si un estudiante obtiene buenos resultados en Biología (Ciencias Naturales), se esperaría que también obtenga buenos resultados en Ciencias Sociales o en Lengua castellana o en Matemáticas. En este mismo sentido se establece la correlación positiva con el nivel de comprensión de lectura de los estudiantes y su rendimiento en los cuatro campos del saber analizados.

Vale aclarar que la existencia de la correlación entre variables no significa necesariamente la presencia de una relación de causa-efecto entre la comprensión lectora y el rendimiento académico. Al aproximarse a la situación de pérdida de asignatura, muchas y disímiles causas pueden ser las razones de bajo rendimiento, así mismo el docente puede dar mayor o menor importancia a la comprensión lectora como criterio de aprobación. Sin embargo, esta correlación en las variables deja abierta la puerta para plantear la necesidad de formar en comprensión lectora y la exigencia de la búsqueda de una educación más integral y de calidad.

En este sentido, investigaciones como la de Gómez, García, Vila, Elosúa y Rodríguez (2014), apoyan la relación de las habilidades de lectura y los logros académicos, principalmente porque entienden la comprensión lectora como una poderosa herramienta de aprendizaje y medio para construir significados y nuevos conocimientos. Así mismo, evidencian la existencia de una correlación entre los procesos propios de la construcción del significado, tales como actualización, conexión e integración de información e inhibición propios de la comprensión lectora y las variables cognitivas superiores: memoria de trabajo (WM) y "uid intelligence". La capacidad de memoria de trabajo (WM) se refiere a la cantidad de elementos que se pueden recuperar durante una tarea compleja. Mientras que la "uid intelligence" es la capacidad de un individuo para razonar y dar solución a problemas novedosos.

En otras palabras, estos autores encuentran que las habilidades de comprensión lectora se vinculan con los procesos ejecutivos de la memoria de trabajo, demostrando que los estudiantes que tienen una puntuación baja en las medidas de la memoria de trabajo (WM) suelen tener un rendimiento por debajo de los estándares esperados en diferentes áreas académicas (Lenguaje y Matemáticas). Es decir, en sus resultados encontraron que los procesos ejecutivos y la comprensión de lectura contribuyeron de forma independiente para explicar una cantidad relevante de la varianza en las medidas de logro académico de los estudiantes. De esta manera concluyen que los procesos ejecutivos de la memoria de trabajo tienen capacidad de predecir el nivel de comprensión lectora y guardan correlación con el desempeño académico. 
Tal como se evidencia en la revisión de la literatura y en los resultados del presente trabajo, la comprensión de texto es una tarea cognitiva altamente exigente que implica el proceso simultáneo de extracción y construcción de significado (Van Dijk \& Kintsch, 1983; Snow \& Sweet, 2003), por lo tanto, la correlación entre los procesos ejecutivos de la memoria de trabajo (WM) y los procesos de comprensión lectora no es inesperada (Gómez et al., 2014). Sin embargo, estos resultados muestran, para la presente investigación, una nueva relación entre comprensión lectora e inteligencia, que puede convertirse en un camino de profundización en cuanto permite ensanchar los horizontes del problema.

En este mismo orden de ideas, Salmerón, García y Vidal-Abarca (2018) abren una nueva puerta, al evaluar los procesos de comprensión lectora en internet frente a los procesos de la memoria de trabajo (WM), por cuanto evidencian que la lectura de internet supone una carga adicional en detrimento de la comprensión, debido a nuevas tareas que asume el lector, tales como la evaluación crítica de contenidos, la navegación, la integración de información de hipertextos, etc. En este sentido, se abren nuevos espacios de reflexión sobre la comprensión y el aprendizaje en el contexto de la sociedad del conocimiento, caracterizados porque van más allá de la escuela y se extienden a lo largo de la vida de los sujetos.

\section{Referencias}

Altman, D. A. (1997). Practical Statistics for Medical Research. London: Chapman \& Hall.

Avendaño, G. S. (2016). La lectura crítica en educación básica secundaria y media: la voz de los docentes. Cuadernos de Lingüística Hispánica, (28), 207-232. http://dx.doi. org/10.19053/0121053X.4916.

Barletta, N. (2001). Intervención metodológica para el desarrollo de las competencias lectoras desde un área particular. En I Coloquio internacionaly III Regional de la Cátedra UNESCO para la lectura y la escritura en América Latina. Universidad del Valle. Cartagena, Colombia.

Belmonte, E. (2006). Marcos teóricos de las pruebas PISA. Conocimientos y destrezas en Matemáticas, Lectura, Ciencias y Solución de problemas. España: Inecse.

Borrero, L. (2008). Enseñando a leer: teoría y práctica e intervención. Bogotá: Norma.

Caso-Niebla, J., \& Hernández-Guzmán, L. (2007). Variables que inciden en el rendimiento académico de adolescentes mexicanos. Revista Latinoamericana de Psicología, 39(3), 487-501.

Cassany, D. (2006). Tras las líneas: sobre la lectura contemporánea. Barcelona: Anagrama. 
Cisneros, M., Olave, G. \& Rojas, I. (2012). Cómo mejorar la capacidad inferencial en estudiantes universitarios. Educación Educadores, 15(1), 45-61. http://dx.doi.org/10.5294/ edu.2012.15.1.3

Coiro, J. (2003). Comprensión de lectura en internet: ampliando lo que entendemos por comprensión de lectura para incluir las nuevas competencias. Eduteka. Recuperado de http://eduteka.icesi.edu.co/modulos/1/163/180/1

Coll, C. (2005). Lectura y alfabetismo en la sociedad de la información. UOC Papers Revista sobre la Sociedad del Conocimiento, (1). Recuperado de http://www.uoc.edu/uocpapers/1/dt/ esp/coll.pdf

Dawson-Saunders, B. \& Trapp, R.G. (1996). Bioestadística médica. México: El Manual Moderno.

Ferreiro, E. (2002). Pasado y presente de los verbos leer y escribir. México: Fondo de Cultura Económica.

Galindo, A. \& Moreno, L. M. (2014). Formación bilingüe en licenciatura de Lenguas Modernas y comprensión lectora argumentativa. Cuadernos de Lingüística Hispánica, (23), 171-190. https://doi.org/10.19053/0121053X.2345.

Gómez, I. M., García, J. A., Vila, J. 0., Elosúa, M. R. \& Rodríguez, R. (2014). The Dual Processes Hypothesis in Mathematics Performance: Beliefs, Cognitive Reflection, Working Memory and Reasoning. Learning and Individual Differences, (29), 67-73.

Grupo de Investigación DiLeMa. (2008a). Estado del arte de las concepciones sobre prácticas de lectura y escritura en la educación básica y media colombiana. Armenia: Universidad del Quindío.

Grupo de Investigación DiLeMa. (2008b). Propuestas dirigidas a los establecimientos educativos para cualificar los procesos de desarrollo de competencias comunicativas en los estudiantes. Armenia: Universidad del Quindío.

Grupo Heterolalia. (2007). Aportes de una experiencia universitaria en lectura y escritura para la consolidación de una política Institucional. En I Encuentro Nacional de Discusión sobre Políticas Institucionales para el Desarrollo de la Lectura y la Escritura en la Educación Superior. ASCUN y Red Nacional de Discusión sobre Lectura y Escritura en Educación Superior.

Icfes. (2010). Informe evaluaciones internacionales. Colombia en Pisa 2009 síntesis de resultados. Bogotá: Icfes.

Icfes. (2013). Sistema Nacional de Evaluación Estandarizada de la Educación. Alineación del examen Saber 11. Bogotá: Icfes. 
León, J. A. (Coord.). (2003). Conocimiento y discurso: claves para inferir y comprender. España: Pirámide.

Llorens, A. C., Gil, L., Vidal-Abarca, E., Martínez, T., Maña, A. \& Gilabert, R. (2011). Prueba de Competencia Lectora para Educación Secundaria (CompLEC). Psicothema, 23(4), 808817.

López, G. \& Arciniegas, E. (2003). El uso de estrategias metacognitivas en la comprensión de textos escritos. Lenguaje, (31), 118-141.

Martín, A. \& Luna del Castillo J. D. (1993). Bioestadística para las ciencias de la salud. Madrid: Orma.

May, 0. et. al. (2001). Las competencias lectoras en los estudiantes universitarios. En Lectura y escritura para aprender a pensar. I Coloquio Internacional y III Regional de la Cátedra UNESCO para la Lectura y la Escritura en América Latina, Cartagena, Colombia.

Milton, J. S. \& Tsokos, J. 0. (2001). Estadística para biología y ciencias de la salud. Madrid: Interamericana McGraw-Hill.

Moreno, V. (2005). Lectores competentes. Revista de educación, (num. ext), 153-167.

Muñoz, A. E. \& Ocaña, M. (2017). Uso de estrategias metacognitivas para la comprensión textual. Cuadernos de Lingüística Hispánica, (29), 223-244. http://dx.doi. org/10319053/01211053X.n29.2017.5865.

Nieto, J. E. (2006). Estrategias de comprensión del discurso escrito expositivo: evaluación e intervención. Barcelona: Edicions Universitat Barcelona.

OCDE. (2006). La competencia lectora. PISA 2006. Marco de la evaluación. Conocimientos y habilidades en Ciencias, Matemáticas y Lectura. Recuperado de http://siie.tamaulipas. gob.mx/sistemas/docs/Pisa/comprension_lectora_PISA.pdf

Ochoa, 0. 0. \& García, A. M. (2013). La secuencia didáctica como estratégica en la enseñanza del ensayo argumentativo. Cuadernos de Lingüística Hispánica, (19), 199-217.

Ortiz, C. A. (2008). Comprensión y producción de textos argumentativos en estudiantes de básica secundaria. En VII Taller Nacional para la Transformación de la Formación Docente en Lenguaje. Universidad Industrial de Santander, Bucaramanga.

Perilla, A., Rincón, G., Gil, J. S. \& Salas, R. (2004). El mejoramiento de los procesos de comprensión de textos académicos en el ámbito universitario. Lenguaje, (32), 159-182.

Salmerón, L., García, A. \& Vidal-Abarca, E. (2018). The Development of Adolescents' ComprehensionBased Internet Reading Activities. Learning and Individual Differences, (61), 31-39. 
Sánchez, E., García, J. R. \& Rosales, J. (2010). La lectura en el aula. Qué se hace, qué se debe bacer y qué se puede hacer. Barcelona: Graó.

Serna, J. L. \& Díaz, J. E. (2015). Propuesta didáctica para la comprensión crítica en la Universidad La Gran Colombia. Cuadernos de Lingüistica Hispánica, (25), 165-180. https://doi. org/10.19053/0121053X.3376.

Solé, I. (2007). Estrategias de lectura/Reading Strategies. México: Advanced Marketing.

Solé, I. (2012). Competencia lectora y aprendizaje. Revista Iberoamericana de Educación, (59), 43-61.

Sweet, A. P. \& Snow, C. E. (Eds.). (2003). Rethinking Reading Comprehension. Solving Problems in the Teaching of Literacy. New York: Guilford Publications.

Van Dijk, T. A. \& Kintsch, W. (1983). Strategies of Discourse Comprehension. New York: Academic Press. 